\title{
Condición de copa, indicador de salud en árboles urbanos del Bosque San Juan de Aragón, Ciudad de México
}

\section{Crown condition, a health indicator in urban trees of the San Juan de Aragon Park, Mexico City}

\author{
Luz de Lourdes Saavedra-Romerol*, Dionicio Alvarado-Rosales', Patricia Hernández-de la Rosa², \\ Tomás Martínez-Trinidad², Gustavo Mora-Aguilera' y Jaime Villa-Castillo³
}

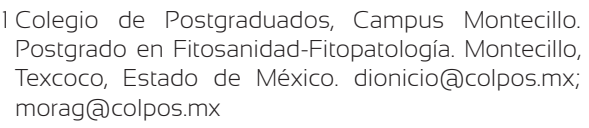

l Colegio de Postgraduados, Campus Montecillo. Postgrado en Fitosanidad-Fitopatología. Montecillo, Texcoco, Estado de México. dionicio(a)colpos.mx; morag@colpos.mx

\author{
2 Colegio de Postgraduados, Campus Montecillo. \\ Postgrado en Ciencias Forestales. pathr@colpos. \\ mx; tomtz@colpos.mx \\ 3 Comisión Nacional Forestal. Sede Guadalajara. Za- \\ popan, Jal. México. jvilla@conafor.gob.mx \\ *Autor de correspondencia. saavedra.luz@colpos.mx
}

\section{RESUMEN:}

Las áreas verdes urbanas aportan diversos servicios a la sociedad y al ambiente. El Bosque San Juan de Aragón, es una de las pocas áreas verdes al noreste de la Ciudad de México y la condición actual de su arbolado en materia de salud no se conoce con precisión. En el presente estudio se implementó y adaptó el protocolo del indicador condición de copa en 28 parcelas circulares de 0.1 ha, establecidas aleatoriamente en el bosque mencionado. A todos los árboles dentro de cada parcela, se les midió el diámetro normal (DN) y altura total (At), se determinó su clasificación taxonómica, condición de copa y densidad de plantación. Para condición de copa, seis variables fueron medidas: proporción de copa viva (Pcv), densidad de copa (Dnc), transparencia de copa (Trc), muerte regresiva (Mrg), posición de copa (Pco) y exposición a la luz (Exl). Las primeras cuatro se midieron en clases de $5 \%$ y las dos restantes a través de una escala ordinal. Se evaluaron 760 árboles, agrupados en 10 familias, 11 géneros y 12 especies. Los valores promedio para las variables de copa fueron, $56.37 \%$ Pcv, 44.38\% Dnc, 4.04\% Mrg y 38.4\% Trc. Solo la Pcv alcanzó los estándares establecidos de salud arbórea encontrados en otros estudios, mientras que las Dnc fueron bajas y los valores de $\operatorname{TrC}$ y Mrg altos.

PAlabras Clave: arbolado urbano, muerte regresiva, proporción de copa, transparencia.

\section{ABSTRACT:}

Green urban areas provide several benefits to society and to the environment. San Juan de Aragon Park, located in the northeast of Mexico City, is one of the few green areas in that urban zone but the health of its trees is not known. In this survey, the crown condition indicator protocol was adapted and applied in 28 circular plots $(0.1 \mathrm{ha})$ established randomly in this park. Normal diameter, total height, species identity, crown condition and tree density were evaluated for all of the trees within these plots. Six crown variables were measured: live crown ratio (Lcr), crown density (Cdn), crown transparency (Ctr), crown dieback (Cdie), crown position (Cpo) and light exposure (Lex). The first four variables were measured in 5\% classes, and the latter two by using an ordinal scale. A total of 760 trees, grouped into 10 families, 11 genera and 12 species, were evaluated. The average values for the crown variables were $56.37 \%$ for Lcr, $44.38 \%$ for Cdn, $4.04 \%$ for Cdie and $38.4 \%$ for Ctr. Only Lcr reached the standards established in other studies, while Cdn was lower and Ctr and Cdie were both higher.

KEY WORDS: green urban areas, crown dieback, live crown ratio, crown transparency.

\section{INTRODUCCIÓN}

En la actualidad, más de la mitad de la población mundial vive en ciudades y esta tendencia irá en aumento en los próximos años (Lorenz, 2005). Para el 2025, la velocidad de urbanización alcanzará los seis billones de personas
(Awal, Ohta, Matsumoto, Toba, Daikoku, Hattori, Hiyama y Park, 2010), lo que ha contribuido a incrementar la necesidad y valor del bosque urbano (Peckham, Duinker y Ordóñez, 2013; Nowak y Walton, 2005), pues en muchos casos la frecuencia de áreas verdes dentro del 
ecosistema urbano disminuye con nuestro estilo de vida (Torrens, 2008). En México, por ejemplo, las áreas verdes han desaparecido a una tasa anual de $3.7 \%$ de acuerdo con Benítez, Chacalo y Barois (1987) y Ezcurra (1996).

En el entorno de una ciudad, el arbolado constituye un recurso invaluable para la sociedad; la belleza escénica y los servicios ambientales que los árboles confieren a los espacios públicos y privados son diversos y, para Johnston (2010), estos beneficios son mayores a los que aporta cualquier otra especie vegetal. Desde el punto de vista ambiental, los árboles impactan en diversos procesos. Altos índices de área foliar tienen mayor influencia en la modificación de ambientes locales (Nowak, 1994), reduciendo el efecto "isla de calor" y regulando la temperatura (Chow y Roth, 2006); además, mejoran la calidad del aire al interceptar contaminantes gaseosos y particulados en sus hojas (Escobedo y Chacalo, 2008; Finlayson y Pitts, 1986). En lo social, el componente arbóreo incrementa el valor patrimonial, disminuye la fatiga mental, la agresión humana y contribuye a mejorar la salud física y psicológica de la población (Escobedo et al., 2008; Nowak, Crane y Stevens, 2006; Todorova, Asakawa y Aikoh, 2004).

A diferencia de árboles que crecen en áreas naturales, el árbol urbano se desarrolla en un entorno más cambiante (Escobedo y Chacalo, 2008; Chacalo, Grabinsky, Vázquez y Aldama, 2005). En general, el árbol urbano presenta un bajo promedio de vida que en muchos casos no excede 25 años (Galvin, 1999), lo anterior, como resultado de la interacción de diversos factores (Bechtold y Randolph, 2009), entre los que destacan: edad, genética de la especie, compactación del suelo (Scharenbroch, Lloyd y Johnson, 2005; Craul, 1999), superficies impermeables (pavimentos y concreto) (Celestian y Martin, 2005), altas temperaturas (Alvarado-Rosales, SaavedraRomero, Fenn, Hernández-Tejeda y Cibrán-Tovar, 2007) y contaminación atmosférica (Alvarado-Rosales y Saavedra-Romero, 2007; Konijnendijk, Nilsson, Randrup y Schipperijn, 2005), restringido espacio vital y, por supuesto, falta de buenas prácticas de manejo. El impacto negativo de estos agentes promueve la presencia de árbo- les enfermos, también reduce su salud y estética (CibriánTovar, Alvarado-Rosales y García-Díaz, 2007), afectando además su arquitectura y estructura, tornando al árbol en elemento de riesgo para los usuarios de las áreas verdes (Angwin, Cluck, Zambino, Oblinger, y Woodruff, 2012).

Aunque la Zona Metropolitana de la Ciudad de México cuenta con numerosas áreas verdes (alamedas, bosques, jardines, parques ecológicos y otras áreas vegetadas), estas no son suficientes debido a la sobrepoblación (Jáuregui-Ostos y Heres-Pulido, 2008), además de que su conservación y manejo no han sido los más apropiados (Escobedo y Chacalo, 2008). Con excepción de algunos casos, la mayoría se encuentran en un estado avanzado de deterioro (Alvarado-Rosales y Saavedra-Romero, 2005); pero no se tienen datos precisos de su condición de vigor y salud actual, por lo que es preciso realizar monitoreos exhaustivos para detectar cambios eventuales o permanentes en el recurso bosque urbano. Esta actividad proveerá información útil para la toma de decisiones en el ámbito político, social y biológico con fines de manejo sustentable (Baker, 1993; McPherson, 1993).

Generalmente, los primeros síntomas de deterioro del árbol se expresan en la apariencia de sus copas (Schomaker et al., 2007); los árboles con altas proporciones de copa viva, diámetros grandes, altas densidades de copa, baja muerte regresiva y transparencia, tienen altas tasas de crecimiento y mayores probabilidades de sobrevivencia (Awal et al., 2010). Las copas densamente foliadas están asociadas con árboles vigorosos, mientras que las pequeñas y de follaje disperso podrían encontrarse en un estado de declinamiento (Shomaker et al., 2007); por lo tanto, a través de su evaluación se podrá determinar su estado de salud actual.

Para evaluar la salud de árboles individuales o del bosque se emplean un conjunto de mediciones, las cuales incluyen: mortalidad arbórea, daños al arbolado, residuos maderables caídos, diversidad arbórea, propiedades físicas y químicas del suelo y condición de copa. El programa de salud forestal en Estados Unidos, es el único inventario nacional que evalúa la condición de copa (Metzger y Oren, 
2001). El protocolo asociado a este indicador se ha descrito en diversas guías de campo (USDA, 2009; Shomaker et al., 2007), sin embargo, requiere ser aplicado bajo diferentes condiciones con el fin de ajustarlo.

En 2008, el Bosque de San Juan de Aragón (BSJA) fue declarado área de valor ambiental por el gobierno capitalino y, junto con el Bosque de Chapultepec, conforman $6.5 \%$ de las áreas verdes más importantes de la Ciudad de México. A pesar de esta declaratoria, el bosque está sujeto a una fuerte presión por la afluencia anual de más de 3 millones de visitantes, así como a un deficiente manejo, principalmente por la falta de presupuesto gubernamental (ALDF, 2011).

\section{OBJetIVo}

Con el propósito de ser referente y de contribuir al desarrollo de nuevas alternativas para evaluar la salud de árboles urbanos, el presente estudio tuvo por objetivo adaptar y aplicar la metodología del indicador condición de copa para determinar el estado actual de salud del arbolado del Bosque San Juan de Aragón, Ciudad de México.

\section{MATERIALES Y MÉTODOS}

\section{Área de estudio}

El estudio se realizó en el Bosque San Juan de Aragón, localizado al noreste de la Ciudad de México (19²7’32" N y 9904'17' W), en la Delegación Gustavo A. Madero a una altitud promedio de $2240 \mathrm{~m}$. El bosque posee una superficie total de 164 ha de las cuales 114 son áreas verdes. En el área verde total, se seleccionaron al azar 10 secciones identificadas por los caracteres B, C, E, F, G, H, J, K, L y M (Fig. 1); en cada una se aplicó una intensidad de muestreo de $2.5 \%$ (Schreuder, Ernst y Ramírez-Maldonado, 2004), quedando establecidas entre 1 y 6 parcelas de muestreo circulares de 0.1 ha $\left(1000 \mathrm{~m}^{2}\right)(\mathrm{r}=17.84 \mathrm{~m})$ por sección (28 parcelas totales). Cada parcela se geoposicionó y todos los árboles dentro de los límites de las mismas se numeraron en dirección de las manecillas del reloj, iniciando por el norte. El marcaje se realizó con etiquetas laminadas de $3.5 \mathrm{~cm} \times 5 \mathrm{~cm}$.

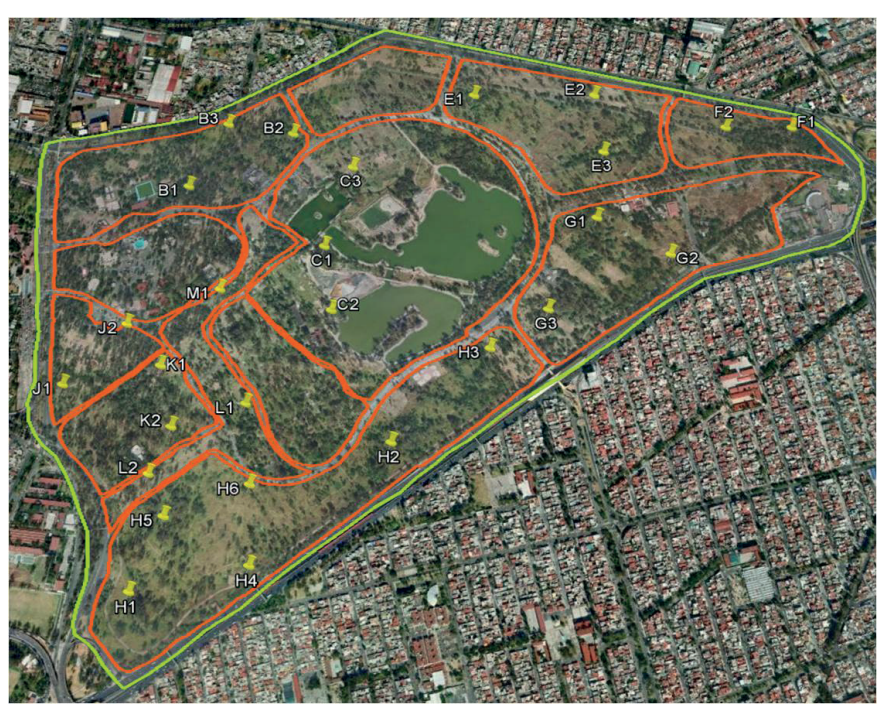

Figura 1. Puntos de muestreo en el Bosque de San Juan de Aragón, Ciudad de México (Imagen de Google Earth, 2014).

\section{Protocolo de medición}

En campo se midieron variables absolutas (Schomaker et al., 2007) en forma visual por una cuadrilla de dos personas, ubicadas a una distancia proporcional a la altura del árbol de interés (Bechtold y Randolph, 2009; Zarnoch, Bechtold y Solte, 2004). Las seis variables para determinar la condición de copa fueron:

Proporción de copa viva ( $\mathrm{Pcv})$, definida como la relación entre el largo de copa y la altura total del árbol (Schomaker et al., 2007; Westfall, Bechtold y Randolph, 2009).

Densidad de copa (Dnc), es un índice de biomasa de copa y representa la cantidad de todos los componentes de la copa incluyendo follaje, ramas y estructuras reproductivas (Randolph, 2006; Bechtold, Randolph y Zarnoch, 2008).

Transparencia de copa ( $\operatorname{Trc})$, estima cuan denso es el follaje sobre las ramas. Mide la cantidad de luz que penetra través de la copa viva (Metzger y Oren, 2001). Trc y Dnc fueron evaluados con la tarjeta densidad-transparencia propuesta por Shomaker et al., (2007).

Muerte regresiva $(\mathrm{Mrg})$, es una medida de la extensión de ramillas defoliadas en la periferia de la copa superior y progresa hacia abajo (Bechtold y Randolph, 2009). Esta variable indica pérdida de vigor o de crecimiento potencial (Metzger y Oren, 2001). 
Posición de copa (Pco), entendida como la posición vertical de cada individuo dentro de cada parcela (Zarnoch et al., 2004), se registró con valores de 1 a 3 para árboles dominantes, codominantes y suprimidos, respectivamente.

Exposición a la luz (Exl), mide la cantidad de luz del sol que recibe el árbol y se calificó con valores de 0 a 5 dependiendo del número de caras del árbol que la recibían (Bechtold, 2003; Zarnoch et al., 2004).

Las primeras cuatro variables se evaluaron en incrementos de $5 \%$ y se registraron en códigos de dos dígitos $(00,05,10 \ldots 99)$. Cada código representa el límite superior de la clase, por ejemplo $1 \%$ a $5 \%$ fue registrado como código 05 (Randolph, Rose, Oswalt y Brown, 2013). El levantamiento de la información se realizó durante la estación de crecimiento junio-agosto del 2013.

\section{Análisis de datos}

Se creó una base de datos en Excel y se analizó estadísticamente con el programa SAS® (Statistical Analysis System) Versión 9.4. Para determinar la distribución de los datos se realizaron pruebas de normalidad Shapiro-Wilk cuando $\mathrm{n} \leq 2000$ con $\alpha=0.05$. La normalidad se comprobó para los valores brutos en porcentaje, así como para su conversión a través de la función arcoseno; finalmente se tomó la decisión de trabajar los datos en porcentaje empleando un análisis paramétrico. Además, se realizó una comparación de medias con la prueba DSH (diferencia significativa honesta de Tukey) y un $\alpha=0.05$.

\section{RESULTADOS}

Se evaluaron 760 árboles y se determinó una mortalidad de $0.78 \%$. La población evaluada representó a 10 familias, 11 géneros y 12 especies. Casuarinaceae, Proteaceae y Cupressaceae fueron las familias con mayor frecuencia con $23.60 \%, 17.50 \%$ y $17.24 \%$, respectivamente. El diámetro normal (DN) y la altura (At) se distribuyeron en tres categorías. Los DN dominantes incluyeron árboles de $1 \mathrm{~cm}$ a $22.7 \mathrm{~cm}$, lo que representó $74 \%$ del total (Fig. 2A); para At, las categorías de $4 \mathrm{~m}$ a $14.8 \mathrm{~m}$ fueron comunes (90.05\%) (Fig. 2B). La densidad de árboles por parcela osciló entre 12 y 57 individuos, resultando en una densidad de plantación de 120 a 570 árboles por hectárea. El DN y At mostraron diferencias significativas $(p<0.0001 \mathrm{y}$ $\alpha=0.05$ ) entre especies, con los valores más bajos para $C$. sempervirens y los mayores para E. camaldulensis (Tabla $1)$.

Tabla 1. Valores promedio de variables dasométricas para 753 árboles evaluados por especie, en el Bosque de San Juan de Aragón, Cd. de México.

\begin{tabular}{lccc}
\hline Especie & $\mathrm{N}^{+}$ & $\mathrm{DN}(\mathrm{cm})$ & $\mathrm{At}(\mathrm{m})$ \\
\hline Eucalyptus camaldulensis Dhnh. & 91 & $31.15^{\mathrm{a}}$ & $15.66^{\mathrm{a}}$ \\
Tamarix gallica L. (H) & 23 & $28.58^{\mathrm{a}}$ & $11.38^{\mathrm{abc}}$ \\
Schinus molle Linn. & 73 & $26.62^{\mathrm{ab}}$ & $8.78 \mathrm{~b}^{\mathrm{abc}}$ \\
Casuarina equisetifolia Forst. & 178 & $26.11^{\mathrm{ab}}$ & $13.28^{\mathrm{ab}}$ \\
Ligustrum lucidum Ait. & 6 & $12.76^{\mathrm{ab}}$ & $7.27^{\mathrm{bc}}$ \\
Acacia retinodes Schlecht. & 57 & $12.63^{\mathrm{ab}}$ & $5.98^{\mathrm{bc}}$ \\
Cupressus lusitanica Klotsch. & 103 & $10.90^{\mathrm{ab}}$ & $6.62^{\mathrm{bc}}$ \\
Fraxinus uhdei (Wenz.) Lingelsh. & 61 & $10.38^{\mathrm{ab}}$ & $5.87^{\mathrm{bc}}$ \\
Grevillea robusta Cunn. & 130 & $9.21^{\mathrm{ab}}$ & $7.49^{\mathrm{bc}}$ \\
Cupressus sempervirens L. & 29 & $6.43^{\mathrm{b}}$ & $4.15^{\mathrm{c}}$ \\
\hline
\end{tabular}

•N: número de árboles; DN: diámetro normal; At: altura total. abc Letras iguales no son significativamente distintas $(\mathrm{p}<\mathrm{O} .001)$

\section{Análisis general}

El análisis de las variables de copa se realizó para todos los árboles vivos (753 árboles) y por separado para las distintas especies de angiospermas y gimnospermas. En el primer caso, se presentan histogramas de frecuencia y frecuencia acumulada, los cuales muestran que la Pcv osciló entre $5 \%$ y $95 \%$ (Fig. 3A), la Dnc de $5 \%$ a $90 \%$ (Fig. 3B), mientras que la Trc y la Mrg (Fig. 3CD) presentaron un rango similar de $0 \%$ a $90 \%$. En lo que respecta a la posición de copa, $47.34 \%$ de los árboles exhibieron una posición suprimida y $36.07 \%$ codominante; en menor número se encontraron árboles dominantes (16.58\%) (Fig. 3E). Árboles con menor exposición a la luz (0 y 1) representaron $44.03 \%$, lo que coincide con la posición suprimida 


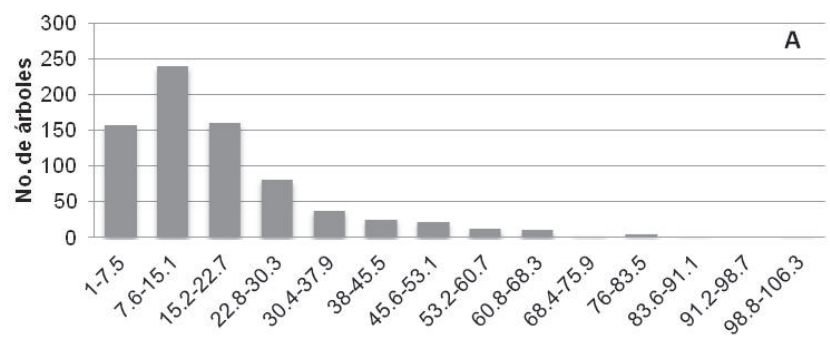

Clases diamétricas $(\mathrm{cm})$

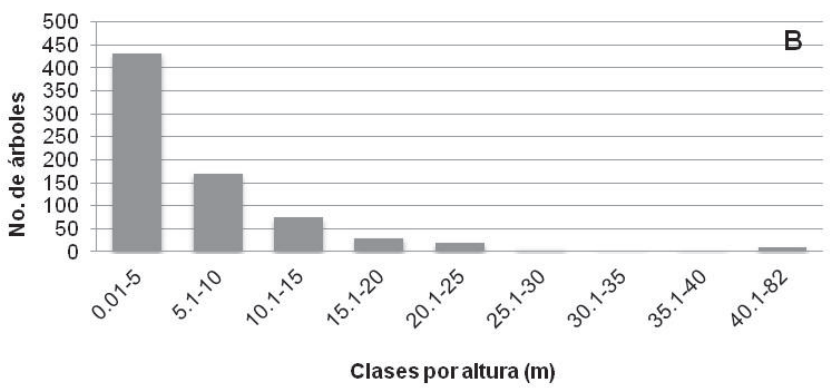

Figura 2. Distribución de categorías diamétricas (A) y altura (B) en los 753 árboles evaluados en el Bosque de San Juan de Aragón, Cd. de México.

que ocupaban. Mientras que los árboles con exposición 4 y 5 son un reflejo de árboles dominantes con $24.66 \%$ (Fig. $3 \mathrm{~F})$.

A pesar de que las Pcv, Dnc y Trc se distribuyen en un intervalo amplio (Tabla 2), la mayoría de las observaciones se distribuyeron en una pequeña porción (Figs. 3ABC). Este comportamiento se confirmó a través de los rangos intercuartiles ( 25 y 75), utilizados como una medida de la dispersión y cuya amplitud para las tres variables fue de
20\%. La amplitud en el caso de la Mrg fue menor (5\%). Finalmente, los histogramas de frecuencia indicaron que fue bajo el número de árboles con altas proporciones de copa viva y densidad, así como altas transparencias de copa (Randolph, 2006).

\section{Por especie}

Los valores promedio entre coníferas fueron significativamente diferentes. La mayor Pcv y Dnc fue para Cupressus sempervirens, mientras que la Trc fue más alta en C. lusitanica. La muerte regresiva no mostró diferencias entre las dos especies de cedro (Tabla 3).

En angiospermas, la Pcv más alta fue para Grevillea robusta con $60.19 \%$ y menor para Fraxinus ubdei y Ligustrum lucidum con $48.61 \%$ y $32.50 \%$, respectivamente (Fig. 4A). La Dnc fue significativamente más alta en Acacia retinodes con $47.19 \%$ y menor en Tamarix gallica con 35\% (Fig. 4B). La Trc más baja fue para Acacia y Ligustrum, las cuales fueron significativamente diferentes al resto de las especies (Fig. 4C). El promedio más alto de muerte regresiva fue para L. lucidum con $17.50 \%$ y la más baja para Acacia, Fraxinus y Grevillea. En general, la condición de copa respecto a densidad, transparencia y muerte regresiva tiende a ser mejor en coníferas (Tabla 3) que en angiospermas (Fig. 4).

\section{Por sección}

Para resaltar diferencias espaciales, se realizó una comparación entre secciones (Tabla 4). Con excepción de Dnc,

Tabla 2. Estadísticos descriptivos de las variables absolutas de copa para los 753 árboles evaluados en el Bosque de San Juan de Aragón, Cd. de México.

\begin{tabular}{lcccccccccccc}
\hline Variable* & $N$ & Media & Asimetría & Curtosis & Min & \multicolumn{4}{c}{ Percentiles } & \multicolumn{3}{c}{ Max } \\
\cline { 7 - 10 } & & & & & & 10 & 25 & 50 & 75 & 90 & \\
\hline Pcv & 754 & 56.37 & -0.27 & 0.04 & 5 & 35 & 45 & 60 & 65 & 75 & 95.00 \\
Dnc & 754 & 44.38 & 0.40 & 0.73 & 5 & 30 & 35 & 45 & 55 & 65 & 90.00 \\
Trc & 754 & 33.84 & 0.32 & -0.02 & 0 & 15 & 25 & 35 & 45 & 55 & 90.00 \\
Mrg & 754 & 4.04 & 4.12 & 21.76 & 0 & 0 & 0 & 0 & 5 & 15 & 90.00 \\
\hline
\end{tabular}

•Pcv: proporción de copa viva; Dnc: densidad de copa; Trc: transparencia de copa; Mreg: muerte regresiva; N: número de árboles. 


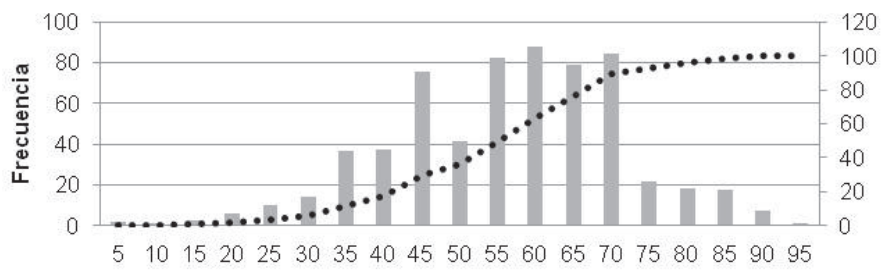

$\%$ Proporción de copa viva

Frecuencia ......\% Acumulado

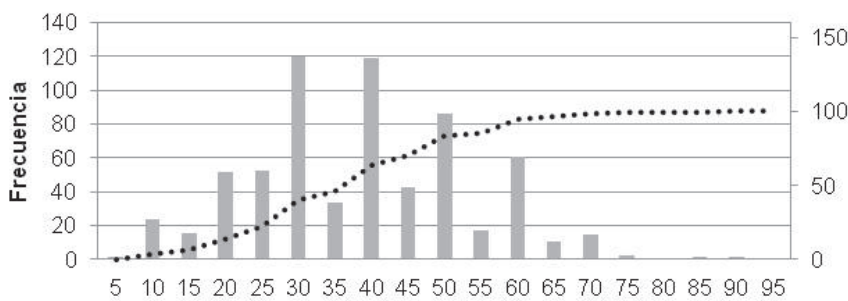

Transparencia de copa $(\%)$

Frecuencia

....... \% Acumulado

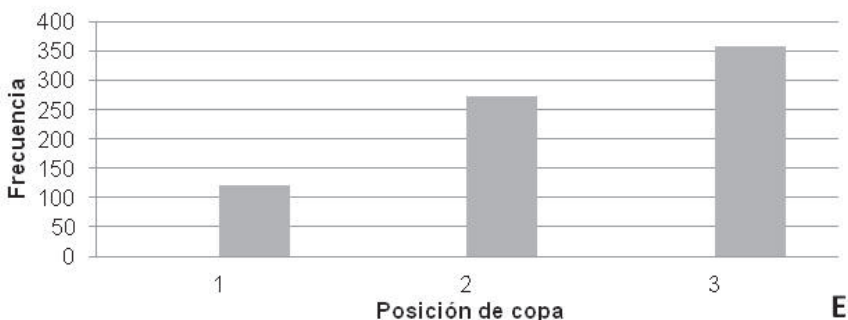

A
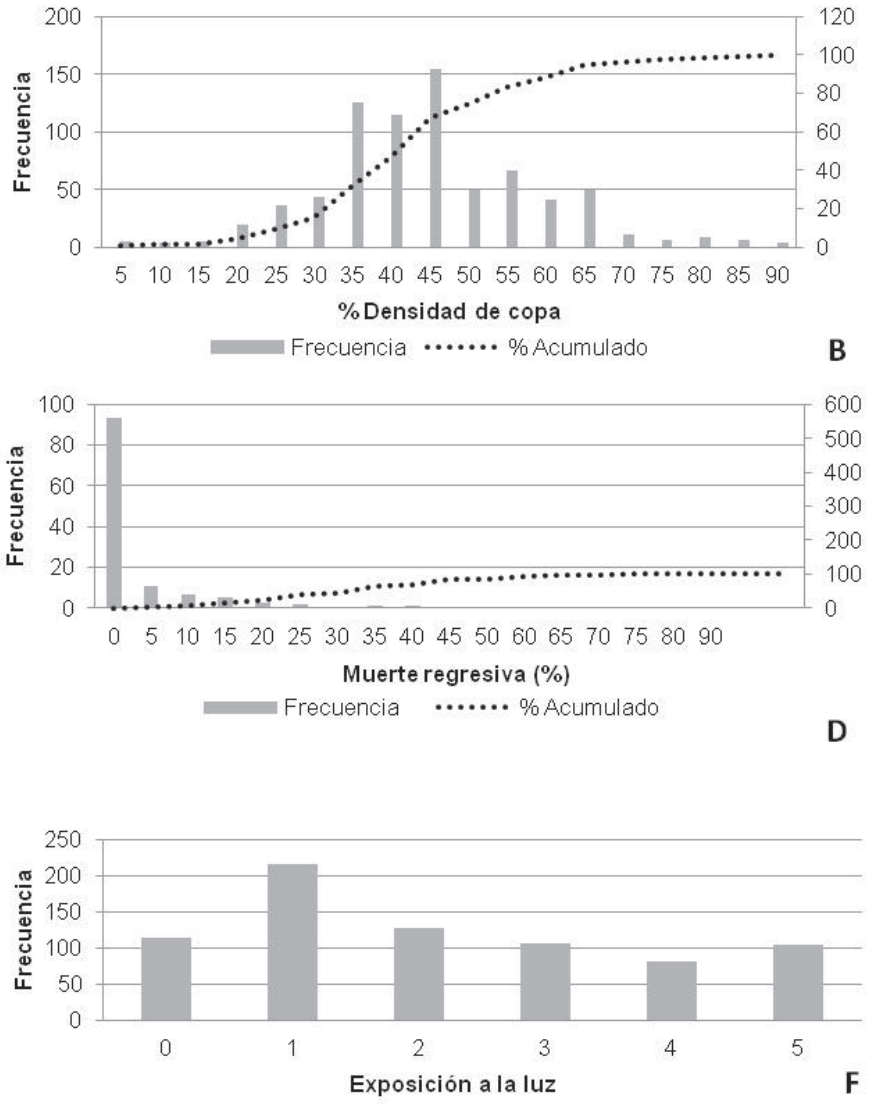

FiguRa 3. Histogramas de frecuencia y frecuencia acumulada para las variables absolutas de copa en los árboles evaluados en el Bosque San Juan de Aragón, Cd. de México.

A. Proporción de copa viva. B. Densidad de copa. C. Transparencia de copa. D. Muerte regresiva. E. Posición de copa y F. Exposición a la luz.
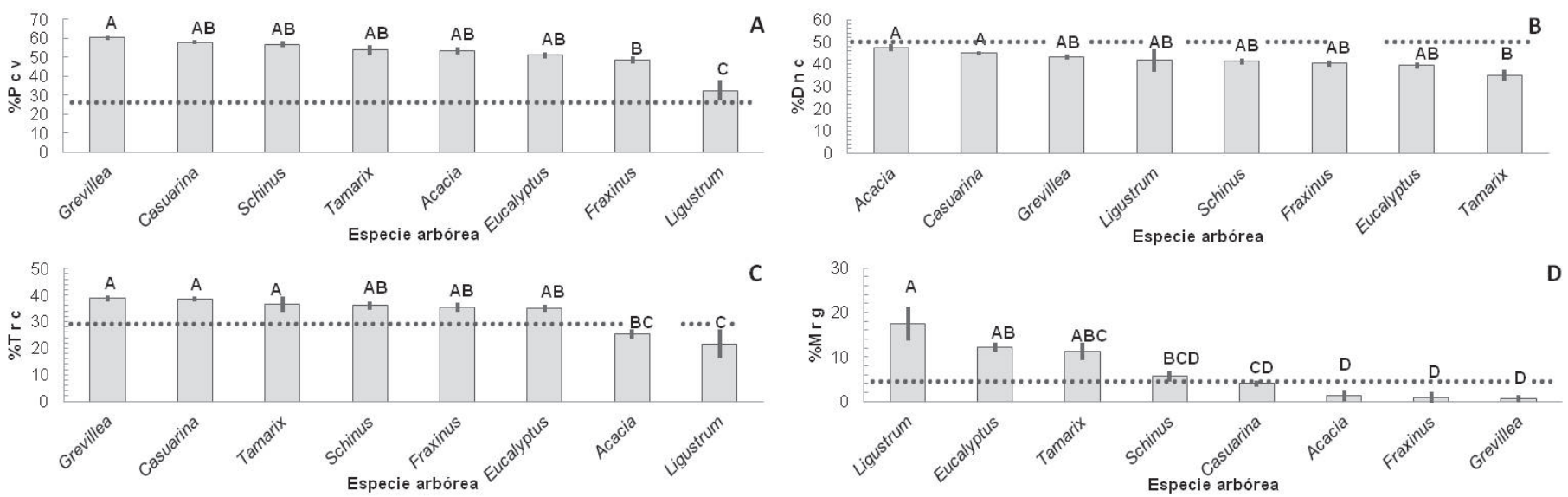

Figura 4. Comparación de variables de copa por especie y error estándar asociado en angiospermas.

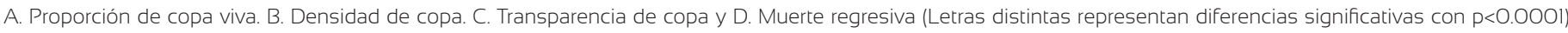
(Línea horizontal punteada indica promedios estándar obtenidos en otros estudios). 
TABla 3. Comparación de variables de copa para las dos especies de coníferas presentes en el Bosque San Juan de Aragón, Cd. de México.

\begin{tabular}{lcccc}
\hline Especie & $\mathrm{Pcv}^{*}$ & $\mathrm{Dnc}^{*}$ & Trc & Mrg \\
\hline Cupressus sempervirens & $68.62 \mathrm{a}$ & $66.55 \mathrm{a}$ & $12.24 \mathrm{a}$ & $1.21 \mathrm{a}$ \\
Cupressus lusitanica & $58.01 \mathrm{~b}$ & $48.45 \mathrm{~b}$ & $26.65 \mathrm{~b}$ & $2.04 \mathrm{a}$ \\
\hline
\end{tabular}

*Letras similares no son significativamente diferentes. Pcv ( $p=0.0026)$; Dnc y $\operatorname{Trc}(\mathrm{p}<\mathrm{O} .0 \mathrm{OOI})$. PCv: proporción de copa viva; Dnc: densidad de copa; Trc: transparencia de copa; Mrg: muerte regresiva.

las variables restantes fueron significativamente diferentes ( $p>0.0001)$. La mayor Pcv se presentó en las secciones E-C y la menor en B. Para Dnc, las 10 secciones tuvieron un promedio menor a $50 \%$. En seis secciones, la Trc fue mayor a $30 \%$, con un intervalo de $32.42 \%$ a $41.84 \%$ y en el caso de Mrg las secciones J y C presentaron porcentajes mayores a $5 \%$.

La Poc influye el valor promedio de Trc y Mrg (Fig. $5)$. Diferencias significativas $(\mathrm{p}<0.001)$ indican un incremento en la Trc, siendo mayor en árboles dominantes y codominantes con valores que oscilaron entre $34.30 \%$ a
TABla 4. Comparación de variables de copa por sección en el Bosque San Juan de Aragón, Cd. de México.

\begin{tabular}{ccccc}
\hline Sección & Pcv (\%) & Dnc (\%) & Trc (\%) & Mrg (\%) \\
\hline C & $65.07 a$ & $43.63 a$ & $34.79 a b c$ & $7.05 a b$ \\
E & $62.48 a b$ & $47.78 a$ & $27.73 c d$ & $3.35 a b$ \\
G & $59.7 a b c$ & $47.5 a$ & $24.57 d$ & $4.09 a b$ \\
H & $56.78 a b c d$ & $46.36 a$ & $32.46 \mathrm{bcd}$ & $3.39 a b$ \\
K & $55.05 a b c d$ & $46.32 a$ & $41.84 a$ & $0.68 b$ \\
L & $53.94 b c d$ & $39.23 a$ & $27.79 c d$ & $3.94 a b$ \\
F & $53.7 b c d$ & $42.39 a$ & $33.91 a b c$ & $3.59 a b$ \\
M & $53.08 \mathrm{bcd}$ & $39.23 a$ & $27.31 c d$ & $4.62 a b$ \\
J & $52.09 c d$ & $41.27 a$ & $39.55 a b$ & $8.66 a$ \\
B & $50.13 d$ & $41.89 a$ & $39.20 a b$ & $4.12 a b$ \\
\hline
\end{tabular}

Medias con letras iguales no son significativamente diferentes ( $p>0.0001$ ).

$35.37 \%$, en tanto que para los suprimidos, fue más bajo, $31.90 \%$. Por su parte, los árboles dominantes presentaron mayor muerte descendente con $10.53 \%$ y, para codominantes y suprimidos, de $2.14 \%$ a $3.65 \%$.
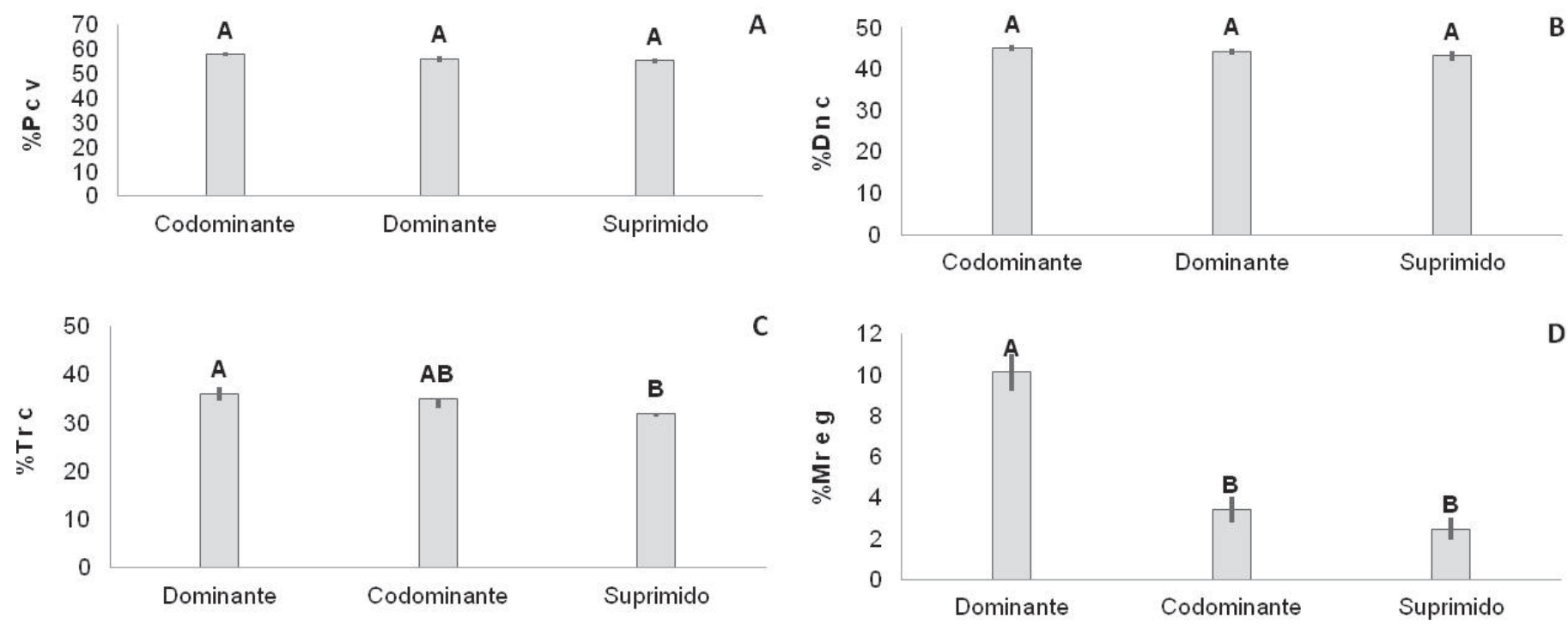

Figura 5. Valores promedio de variables de copa y error estándar con base en la posición de copa de los árboles evaluados en el Bosque San Juan de Aragón, Cd. de México. 
La exposición a la luz (Exl) tuvo influencia únicamente sobre la medidas de abundancia foliar, es decir, la densidad y la transparencia (Fig. 6). En el caso de Dnc, los árboles con menor exposición a la luz (0 y 1 caras), presentaron también las menores densidades de copa, con un intervalo de $39.06 \%$ a $41.21 \%$. Las exposiciones 2 a 5 fueron significativamente mayores oscilando entre $45.14 \%$ a $48.06 \%$. En lo que se refiere a Trc, los árboles con una Exl de 1, mostraron copas más ralas, $35.64 \%$, que las de $\mathrm{Exl}=4$, con $29.81 \%$.

\section{DISCUSIÓN}

El monitoreo extensivo e intensivo del indicador condición de copa en escenarios forestales y urbanos de Estados Unidos de América ha permitido generar estándares de salud arbórea a nivel de especie y de sitio. En términos generales, se considera como saludables a los árboles poseedores de las siguientes características: (a) Proporción de copa viva $>30 \%$; (b) Densidad de copa $>50 \%$; (c) Transparencia $<30 \%$ y (d) Muerte regresiva $<5 \%$ (Randolph, 2013, 2004; Bechtold et al., 2008; Metzger y Oren, 2001).

Con base en los estándares citados, la comunidad arbórea, sus grupos (coníferas y angiospermas) y especies evaluadas en el BSJA, presentaron $\mathrm{Pcv}_{\mathrm{s}}$ superiores a $30 \%$
(Tabla 3; Fig. 4A: línea de corte eje "Y”). Por sí sola, la proporción de copa viva no fue apta para expresar ni abundancia de follaje ni su arreglo espacial dentro de la copa, por lo que, para obtener un panorama completo sobre el estado de salud de los árboles, se analizaron de forma general y por especie, la Dnc, Trc y Mreg.

La especie Cupressus sempervirens fue la única en superar el estándar de Dnc establecido, es decir, > 50\% (Tabla 3), el resto de las especies, una conífera y ocho latifoliadas presentaron densidades de copa menores (Fig. 4B, línea de corte eje "Y"). A pesar de que la Dnc puede variar entre especies, edades, genotipos y periodo de evaluación (Zaragoza-Hernández, Cetina-Alcalá, López-López, Chacalo-Hilú, de la Isla y Gonzáles-Rosas, 2014; Cumming, Galvin, Rabaglia, Cumming y Twardus, 2001), se ha comprobado que árboles con copas densas crecen con mayor celeridad en diámetro que los árboles que poseen copas dispersas, por ello, dicha variable junto con la transparencia son componentes clave que se han empleado en diferentes sistemas de evaluación de salud arbórea (Dobbertin, 2005; Belanger y Anderson, 1992). Los estudios de Steinman (2000), indican que tanto coníferas como angiospermas con densidades menores a $30 \%$ tienen mayores posibilidades de morir en el lapso de un año. La identificación oportuna de individuos con tales caracterís-
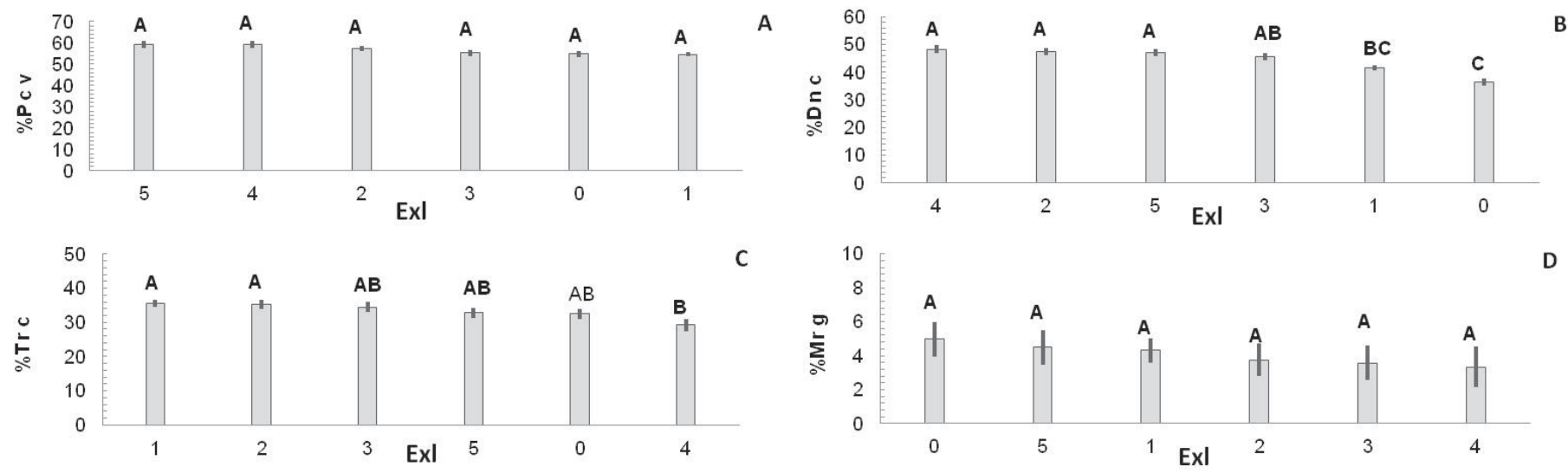

Figura 6: Valores promedio de variables de copa y error estándar con base en la exposición de la copa a la luz para los árboles evaluados en el Bosque San Juan de Aragón, Cd. de México. 
ticas dentro del bosque urbano, permitiría implementar actividades de manejo dirigidas a mejorar las condiciones de crecimiento de los árboles afectados, o en su defecto, establecer programas de saneamiento destinados a reducir el riesgo sobre los usuarios del recurso bosque.

Con excepción de Acacia y Ligustrum, las especies restantes tienen valores altos de transparencia de copa, que superan $30 \%$ establecido inicialmente (Fig. 4C), lo que indica que estos árboles están creciendo en condiciones de moderado a severo estrés (Metzger y Oren, 2001; Millers, Durham, Anderson, Burkman, Radnor y Hoffard, 1992). De acuerdo con Winn, Araman y Sang-Monk, (2011), un incremento en Trc de un año a otro puede ser indicativo de un árbol fuertemente estresado, lo que va en detrimento de su crecimiento potencial, sobrevivencia e incluso de su potencial reproductivo. Diferentes causas pueden incrementar la transparencia de copa, pero los más comunes incluyen insectos defoliadores, enfermedades, contaminación atmosférica, compactación de suelo, disminución de tamaño foliar y pérdida de ramas (Shomaker et al., 2007).

Los árboles con mayor muerte regresiva mostraron copas pobres, follaje disperso y abundancia de ramillas muertas en copa superior. Tamarix, Eucalyptus y Ligustrum fueron las especies con Mrg superiores a 5\%, calificándose como no saludables (Metzger y Oren, 2001) (Fig. 4D). La presencia de factores externos entre ellos extensos periodos de sequía, suelos inundados y patógenos de raíz son posiblemente responsables de muerte prematura de ramas (Brooks, Miller y Burkman, 1991; Nadolny, 1995) y en el BSJA, al menos los dos primeros factores fueron frecuentes.

El estudio de Zaragoza-Hernández et al., (2014) en tres alamedas de la Ciudad de México, concluyen que las especies más destacadas por su salud de copa fueron Cupressus sempervirens, Ligustrum lucidum, Populus alba y Fraxinus uhdei. La Dnc de C. sempervirens fue de $62.19 \%$, ligeramente más baja a la estimada para el BSJA. Por su parte, la Mrg de $3.75 \%$ y $\operatorname{Trc}$ de $29.33 \%$ fueron considerablemente mayores. Si bien, los valores promedio de estas especies son distintos a los del presente estudio, es posible que las condiciones climáticas (distintas unidades ambientales respecto a precipitación, temperatura, niveles de contaminación, etc.) y de sitio (relieve, tipo de suelo) (Gobierno del Distrito Federal, 2001), estén influyendo en los resultados obtenidos.

Se sabe que la estructura de un árbol, está determinada por el tamaño, forma y la fisiología de su copa (Ford, 1985). Las copas colectan, producen y compiten por recursos, especialmente con árboles vecinos, pero quizás la competencia por espacio de crecimiento y radiación son las más importantes, ya que determinan el adecuado crecimiento y buen desarrollo de un árbol. Para Rouvinen y Kuuluvainen (1997), el incremento en biomasa está determinado por la cantidad y calidad de luz interceptada por las copas de los árboles. En el presente estudio, se observó que los árboles con mayor número de caras expuestas a la luz (2 a 5), presentaron también mayores densidades de copa (Fig. 6B), a diferencia de los menos expuestos $(0$ caras) y que se ubicaron en la categoría de árbol suprimido.

Por su parte, los árboles dominantes y codominantes presentaron mayores valores de transparencia y muerte regresiva (Fig. 5). Se presume que, al ser árboles de una madurez mayor, su tiempo de exposición a distintos factores de estrés ha menguado considerablemente su salud, comparado con los árboles suprimidos. En adición, cuando una copa crece, una proporción creciente de su volumen está ocupado por un núcleo que soporta ramas sin follaje. Esta estructura contribuye muy poco con fotosintatos, sin embargo sí los utiliza para la producción de madera y para la respiración (Ford, 1985). La elevada demanda de fotosintatos para soportar las copas de árboles dominantes y suprimidos podría explicar también su mayor transparencia y muerte regresiva debido a una demanda no satisfecha.

La copa es el principal componente de la productividad primaria. Sus dimensiones, formas y distribución de su biomasa (ramas y follaje) pueden reflejar el estado general de salud del árbol (Cumming, Nowak, Twardus, Hoehn, Mielke y Rideout, 2007; Zarnoch et al., 2004). Copas simétricas y densamente foliadas están asociadas a 
un alto potencial fotosintético (Randolph, 2004, 2013; Awal et al., 2010), mientras que las copas pequeñas y dispersas apuntan a condiciones desfavorables de crecimiento (pobres condiciones de sitio, enfermedades, competencia, estrés hídrico, compactación de suelo, estrés crónico, etc.) (Zarnoch et al., 2004) y son típicas de árboles en declinación (Nadolny, 1995). La apariencia y condición respecto a la cantidad y distribución del follaje proporcionan una idea clara de la historia de vida de cada individuo y del ambiente donde se está desarrollando.

\section{CONCLUSIONES}

El indicador condición de copa y sus variables absolutas aportaron información confiable sobre el estado de salud actual del arbolado del Bosque San Juan de Aragón, Ciudad de México, específicamente, para la estación de crecimiento julio-agosto 2013. La densidad de las copas y sus transparencias permitieron obtener un panorama general sobre los porcentajes de biomasa presente en las copas de los árboles evaluados; mientras que a través de la muerte regresiva se identificaron árboles con declinamiento prematuro. En general, el estado de salud de los árboles de este bosque urbano se calificó de buena a pobre debido a las altas proporciones de copa viva, bajas densidades de copa y altas transparencias de copa. Se requiere de investigación adicional para identificar los diferentes agentes de estrés que afectan a las especies con una pobre condición de copa.

El indicador condición de copa, ha sido ampliamente usado con el propósito de identificar cambios y tendencias en el recurso bosque urbano. En México, es posible implementar y extender el uso de esta metodología para determinar la salud actual de sus áreas verdes arboladas.

\section{REFERENCIAS}

ALDF (2011). Rescatar bosque de Aragón debe ser prioridad. ALDF-Asamblea legislativa del Distrito Federal. Recuperado de http://aldf.gob.mx/comsoc-rescatar-bosque-aragon-debe-ser-prioridad-8503.html.

Alvarado-Rosales, D., Saavedra-Romero, L. de L., Fenn, M., Hernández-Tejeda, T. y Cibrián-Tovar, D. (2007). Agentes abióticos, causantes de estrés y muerte/Abiotic factors that causes stress and death. En T. D. Cibrián, R. D. Alvarado y S.E. García D. (Eds.), Enfermedades forestales en México/Forest diseases in Mexico (pp. 11-41). Chapingo, México: Universidad Autónoma Chapingo.

Alvarado-Rosales, D. y Saavedra-Romero, L. de L. (2007). Declinación del oyamel/Fir decline. En T.D. Cibrián, R.D. Alvarado y S.E. García D. (Eds.), Enfermedades forestales en México/Forest diseases in Mexico. Universidad Autónoma Chapingo; CONAFOR-SEMARNAT, México; Forest Service USDA, EUA; NRCAN Forest Service, Canadá y Comisión Forestal de América del Norte, COFAN, FAO. Chapingo, México. p. 444-447.

Alvarado-Rosales, D. y Saavedra-Romero, L. L. (2005). El género Cladocolea (Loranthaceae) en México: muérdago verdadero o injerto. Revista Chapingo Serie Ciencias Forestales y del Ambiente, 11 (1), 5-9.

Angwin, P. A., Cluck, D. R., Zambino, P. J., Oblinger, B. W. y Woodruff, W. C. (2012). Hazard tree guidelines for Forest Service facilities and roads in the Pacific Southwest Region. (Report RO-12-01). Forest Health Protection. 40 p.

Awal, M. A., Ohta, T., Matsumoto, K., Toba, T., Daikoku, K., Hattori, S., Hiyama, T. y Park, H. (2010). Comparing the carbon sequestration capacity of temperate deciduous forests between urban and rural landscapes in central Japan. Urban Forestry and Greening, 9, 261-270.

Baker, F. A. (1993). Monitoring the urban forest: case studies and evaluations. Environmental Monitoring and Assessment, 26, 153-163.

Bechtold, W. A. (2003). Crown position and light exposure -an alternative to field-assigned crown class. Northern Journal of Applied Forestry, 20 (4), 154-160.

Bechtold, W. A., Randolph, K. C. y Zarnoch, S. J. (2008). The power of FIA phase 3 Crown-indicator. En W. Williams, M. Gretchen y R. Czaplewski (Comps.), Forest Inventory and Analysis (FIA) Symposium; October 1-23, 2008 (pp. 1-21). Park City, UT. Proc. RMRS-P-56CD. Fort Collins, CO: U.S. Department of Agriculture, Forest Service, Rocky Mountain Research Station.

Bechtold, W. A. y Randolph, K. C. (2009). The crown-condition indicator. Forest Health Indicators US/Mexico Workshop. Guadalajara, México. April 26-May 01. 
Belanger, R. P. y Anderson, R. L. (1992). A guide for visually assessing crown densities of Loblolly and Shortleaf pines. (Res-Note SE-352). Asheville, NC: U.S. Department of Agriculture Forest Service. Southern Forest Experimental Station. 4 p.

Benítez, G., Chacalo, A. y Barois, I. (1987). Evaluación comparativa de la pérdida de la cubierta vegetal y cambios en el uso del suelo en el sur de la Ciudad de México. En E. Rapoport E. e I. López-Moreno (Eds.), Aportes a la ecología urbana de la Ciudad de México. México: Limusa.

Brooks, R. T., Miller, W. M. y Burkman, W. (1991). Forest Health Monitoring, New England. (NE-INF-94-91) USDA Forest Service, Northeastern Area. 9 p.

Celestian, S. B. y Martin, C. A. (2005). Effects of parking lot location on size and physiology of four southwesthern U.S. landscape trees. Journal of Arboriculture, 31 (4), 191-197.

Cibrián-Tovar, D., Alvarado-Rosales, D. y García-Díaz, S. (Eds.). (2007). Enfermedades forestales en México/Forest diseases in Mexico. Chapingo, México: Universidad Autónoma Chapingo. 587 p.

Chacalo, A., Grabinsky, J., Vázquez, H. J. y Aldama, A. (2005). Threats to urban green areas -case study: Mexico City. En C. C. Konijnendijk, J. Schipperijn y K. Nilsson (Eds.), Urban forests and trees. Proceedings No. 2. (pp. 177188). Luxembourg: Office for Official Publications of the European Communities.

Chow, W. T. L. y Roth, M. (2006). Temporal dynamics of the urban heat island of Singapore. International Journal of Climatology, 26, 2243-2260.

Craul, P. J. (1999). Urban soils. Applications and practices. EUA: John Wiley \& Sons, Inc.

Cumming, B. A., Galvin, M. F., Rabaglia, R. J., Cumming, J. R. y Twardus, D. B. (2001). Forest health monitoring protocol applied to roadside trees in Maryland. Journal of Arboriculture, 27 (3), 126-138.

Cumming, B. A., Nowak, D. J., Twardus, D. B., Hoehn, R., Mielke, M. y Rideout, R. (2007). Urban forest of Wisconsin: Pilot Monitoring Project 2002. (NA-FR-05-07) USDA Forest Service, Northeastern Area. National Forest Health Monitoring Program.
Dobbertin, M. (2005). Tree growth as indicator of tree vitality and of tree reaction to environmental stress: a review. European Journal of Forest Research, 124, 319-333.

Escobedo, F. y Chacalo, A. (2008). Estimación preliminar de la descontaminación atmosférica por el arbolado urbano de la Ciudad de México. Interciencia, 33 (1), 29-33.

Escobedo, F. J., Wagner, J., Nowak, D. J., de la Maza C. L., Rodríguez, M. y Crane, E. D. (2008). Analyzing the cost-effectiveness of Santiago Chile's policy of using urban forests to improve air quality. Journal of Environmetal Management, 86, 148-157.

Ezcurra, E. (1996). De las chinampas a la megalópolis. El medio ambiente en la Cuenca de México. México: FCE.

Finlayson, P. B. J. y Pitts, J. N. (1986). Atmospheric chemistry: fundamental and experimental techniques. E. U. A.: John Wiley \& Sons.

Ford, E. D. (1985). Branching, crown structure and the control of timber production. En M. G. R. Cannel y J. E. Jackson (Eds.), Attributes of trees as crop plants (228-252). Monks Wood, UK.: Institute of Terrestrial Ecology.

Galvin, M. F. (1999). A methodology for assessing and managing biodiversity in street tree population: a case study. Journal of Arboriculture, 25 (3), 124-128.

Gobierno del Distrito Federal. (2001). Manual técnico para el establecimiento y manejo integral de las áreas verdes urbanas del Distrito Federal. Tomo II. México: Secretaría de Medio Ambiente.

Jáuregui-Ostos, E. y Heres-Pulido, M. E. (2008). El clima/bioclima de un parque periurbano de la Ciudad de México. Investigaciones Geográficas, Boletín del Instituto de Geografía, 67, 102-112.

Johnston, M. (2010). Trees in towns II and the contribution of arboriculture. Journal of Urban Forestry, 33 (1), 27-41.

Konijnendijk, C. C., Nilsson, K., Randrup, T. B. y Schipperijn, J. (Eds.). (2005). Urban forest and trees. Países Bajos: Springer.

Lorenz, K. y Kandeler, E. (2005). Biochemical characterization of urban soil profiles from Stuttgart, Germany. Soil Biology \& Biochemistry, 37, 1373-1385.

McPherson, E. G. (1993). Monitoring urban forest health. Environmental Monitoring and Assessment, 26, 165-174. 
Metzger, J. M. y Oren, R. (2001). The effect of crown dimensions on transparency and the assessment of tree health. Ecological Application, 11 (6), 1634-1640.

Millers, I., Durham, N. H., Anderson., R., Burkman, W., Radnor, P. A. y Hoffard, W. (1992). Crown condition rating guide. USDA Forest Service. Northeastern Area and Southern Region.

Nadolny, C. (1995). Causes of tree decline /dieback in NSW. En A. Kater (Ed.), Redressing rural tree decline in NSW: proceedings of the 'After Dieback' conference presented by Greening Australia, Orange Civic Centre (pp. 11-18). Orange, NSW: Greening Australia.

Nowak, D. J., Crane, D. E., y Stevens, J. C. (2006). Air pollution removal by urban trees and shrubs in the United States. Urban Forestry and Urban Greening, 4, 115-123.

Nowak, D. J. y. Walton, J. T (2005). Projected urban growth (2000-2005) and its estimated impact on the US forest resource. Journal of Forestry, 103, 383-389.

Nowak, D. J. (1994). Urban forest structure: the state of Chicagós urban forest. En E. G. McPherson, D. J. Nowak y R. Rowan (Eds.), Chicago's urban forest ecosystem: results of the Chicago urban forest climate Project. (General Technical Report NE-186). Radnor, PA: USDA Forest Service. Northeaster Forest Experiment Station. 201 p.

Peckham, S., Duinker, P. N. y Ordóñez, C. (2013). Urban forest values in Canada: views of citizens in Calgary and Halifax. Urban Forestry and Urban Greening, 12, 154-162.

Randolph, K. C. (2004). An evaluation of change in tree crown characteristics to assess forest health in two Indiana State Parks. Northern Journal of Applied Forestry, 21 (1), 50-55.

Randolph, K. C. (2006). Descriptive statistics of tree crown condition in the Southern United States and impacts on data analysis and interpretation. (Gen. Tech. Rep. SRS94). Asheville, NC: US Department of Agriculture Forest Service, Southern Research Station.

Randolph, K. C. (2013). Development history and bibliography of the U.S. Forest Service crown-condition indicator for forest health monitoring. Environmental Monitoring Assessment, 185, 4977-4993.

Randolph, K. C., Rose, A. K., Oswalt, C. M. y Brown, M. J. (2013). Status of black walnut (Juglans nigra L.) in the eastern United States of the discovery of thousand cankers disease. Southern Appalachian Botanical Society, 78 (1), 2-14.

Rouvinen, S. y Kuuluvainen, T. (1997). Structure and asymmetry of tree crowns in relation to local competition in a natural mature Scots pine forest. Canadian Journal of Forest Research, 27, 890-902.

Scharenbroch, B. C., Lloyd, J. E. y Johnson, J. L. (2005). Distinguising urban environments with physical, chemical and biological soil properties. Pedobiologia, 49, 283-296.

Schomaker, M. E., Zarnoch, S. J., Bechtold, W. A., Latelle, D. J., Burkman, W. G. y Cox, S. M. (2007). Crown-condition classification: A guide to data collection and analysis. (General Technical Report SRS-102) USDA Forest Service.

Schreuder, H. T., Ernst, R. y Ramírez-Maldonado, H. (2004). Statistical techniques for sampling and monitoring natural resources. (General Technical Report RMRSGTR-126). USDA Forest Service.

Steinman, J. (2000). Tracking the health of trees over time on forest health monitoring plots. En M. Hansen y T. Burk (Eds.), Proc. of the IUFRO Conference integrated tools for natural resources inventories in the $21^{\text {st }}$ century (Gen. Tech. Rep. NC-212) (pp. 224-339). USDA For. Serv.

Todorova, A., Asakawa, S. y Aikoh, T. (2004). Preference for and attitudes towards streets flowers and trees in Sapporo, Japan. Landscape and Urban Planning, 69, 403416.

Torrens, P. M. (2008). A toolkit for measuring sprawl. Applied Spatial Analysis, 1, 5-36.

USDA (2009). Forest inventory and analysis national core field guide (Phase 2 and 3), version 4.0. Recuperado de http:// fia.fs.fed.us/library/field-guides-methods-proc/.

Westfall, J. A., Bechtold, A. W. y Randolph, K. C. (2009). Tree crown indicator. En J. A. Westfall (Ed.), FIA National Assessment of data quality for forest health indicators (3-15). (Gen. Tech. Rep. NRS-53). Newtown Square, PA: U.S. Department of Agriculture, Forest Service, Northeastern Research Service.

Winn, M., Araman, P. A. y Sang-Mook, L. (2011). Urban Crowns: an assessment and monitoring tool for urban 
trees. (Gen. Tech. Rep. SRS-135). Asheville, NC: U.S. Department of Agriculture, Forest Service, Southern Research Station.

Zaragoza-Hernández, A., Cetina-Alcalá, V. M., López-López, M. A., Chacalo-Hilú, A., de la Isla, M. L. y GonzálezRosas, H. (2014). General health condition in forest species in three parks in Mexico, City: Crown condition. Research Journal Environmental Sciences, 8 (1), 1-16.

Zarnoch, S. J., Bechtold, W. A. y Solte, K. W. (2004). Using crown condition variables as indicators of forest health. Canadian Journal of Forest Research, 34, 1057-1070.
Manuscrito recibido el 31 de octubre de 2014

Revisado el 25 de enero de 2016

Aceptado el 14 de marzo de 2016

Este documento se debe citar como:

Saavedra-Romero, L. L., Alvarado-Rosales, D., Hernández de la Rosa, P., Martínez-Trinidad, T., Mora-Aguilera, G. y Villa-Castillo, J. (2016). Condición de copa, indicador de salud en árboles urbanos del Bosque San Juan de Aragón, Ciudad de México. Madera y Bosques, 22 (2), 15-27. 